\title{
The effect of rotation upon dental structure components following orthodontic fix appliance
}

\author{
Mariana Păcurar ${ }^{1}$, Ligia Brezeanu ${ }^{1}$, Cristian Olteanu², Karina Dombi
} Dragos Sita ${ }^{4}$, Cristina Molnar ${ }^{1}$

1) Orthodontic Department, Faculty of Dental Medicine, University of Medicine, Pharmacy Science and Technology, Târgu-Mureș, Romania

2) Orthodontic Department, Faculty of Dental Medicine, Iuliu Hatieganu University of Medicine and Pharmacy, Cluj-Napoca, Romania

3) Paediatric Dentistry Department, Faculty of Dental Medicine, "Carol Davila" University of Medicine, Pharmacy, Bucharest, Romania

4) Endodontic Department, Faculty of Dental Medicine, University of Medicine, Pharmacy Science and Technology, Târgu-Mureș, Romania
DOI: $10.15386 / \mathrm{mpr}-1519$

Manuscript received: 05.07.2019

Accepted: 02.09.2019

Address for correspondence:

cristidolteanu@yahoo.com

\begin{abstract}
Background and aims. The purpose of the study is to evaluate through a FEM (Finite Element Method) the effects of the rotation movement upon a complex structure (enamel- pulp -alveolar bone, PDL), for external load.
\end{abstract}

Method. The progressive action of a fixed orthodontic device on three teeth: first molar, first and second premolar is modeled and simulated with the components placed on the buccal and palatal surfaces of the tooth. For the reproduction of a situation similar to the real one, the loading of the model was performed through a nodal force applied at a height of the crown, of various amplitudes, $\mathrm{F}=1 \mathrm{~N} ; 2,3$ and $4 \mathrm{~N}$. The values of stress are: maximum stress of the whole structure and the shearing effect for the pulp.

Results. The characteristics of the material are Young's E modulus and Poisson's ratio of the components of the modeled structure. The most stressed elements of the structure are the pulp and the ligament, revealed by von Mises stress. The elements of the structure are mainly stressed in fiber compression in the direction of the moment's action given by the orthodontic forces and in fiber stretching in the opposite direction. Out of the orthodontic movements: translation, tipping, intrusion-extrusion , rotation is the most dangerous.

Conclusions. The accumulated stress effect in the pulp becomes dangerous. The orthodontic movements given by dental force values higher than $1.5-2 \mathrm{~N}$ are to be avoided.

Keywords: finite element method, rotation, force, stress, displacements

\section{Background and aims}

A rotation is a modification of a singular tooth in which the tooth rotates around its long axis of $90^{\circ}$ that intersects with the tooth in its totality (crown and root). Rotation is a movement that frequently tends to relapse due to the periodontal structures and insufficient use of contention devices.

Finite element analysis is a noninvasive technique for testing a particular design [1], especially the teeth movement induced by orthodontic forces.

The center of rotation is an arbitrary point located at a distance from the center of resistance - the center of mass of the tooth - around which the tooth rotates with the application of a force applied to the crown. The position of the center of rotation never coincides with the position of the center of resistance [2] (Figure 1).

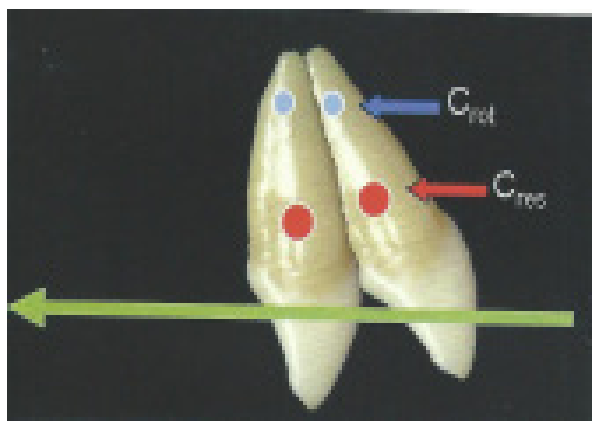

Figure 1. The center of rotation and resistance.

Application of a force upon the $\mathrm{C}_{\text {res }}$ will produce a pure translation and an application of a moment around the $\mathrm{C}_{\text {res }}$ will create a pure rotation. If the force is applied at the level of the crown, this will produce an inclination of the tooth, composed of a translation movement and a rotational one $[3,4]$. 
The center of rotation $\mathrm{C}_{\text {rot }}$ will be determined according to the values of the displacements following the direction of the force, the axis Oy, Syy in the nodes of the vertical axis of the tooth. On a side of the node, with zero displacement in the direction of application of the force, the periodontal fibers will be stretched - positive values of displacements, and on the other side the periodontal fibers will be compressed - negative values of displacements [5]. The position of the center of resistance $\mathrm{C}_{\text {res }}$ and of the center of rotation $\mathrm{C}_{\text {rot }}$ differs depending on the geometry and the morphology of the tooth, but also changes depending on the location of the application of the force, respectively the positioning of the bracket on the tooth.

The purpose of the study is to evaluate through a FEM (Finite Element Method) the effects of the rotation movement upon a complex structure (enamel - pulp alveolar bone, PDL), for external load.

\section{Methods}

Rotation of the root without the displacement of the crown's position is difficult to produce, since the tooth is embedded in the bone with little movement (Figure 2).

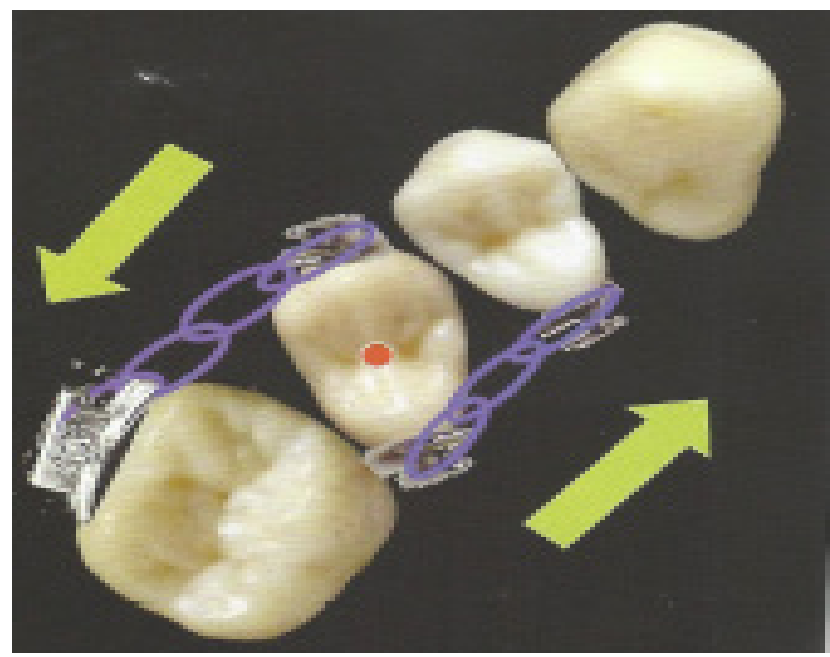

Figure 2. The rotation movement.

The structure modeled and studied is: first molar, premolar 1 and premolar 2, with all its constituents elements: dentine, pulp, periodontal ligament - alveolar bone.

Corresponding to the geometry, dimensions and morphological data in the specialty textbooks of the first, second premolar and first molar, a two-dimensional plane model was created, representing a median section on the height of the structure, perpendicular to the mesial-distal sides, comprising enamel - tooth - PDL-alveolar bone pulp (Figure 3). The used finite elements for the plane model created are bidimensional, 2D-type.
The progressive action of a fixed orthodontic device on three teeth: first molar, first and second premolar is modeled and simulated with the components placed on the buccal and palatal surfaces of the tooth.

For the reproduction of a situation similar to the real one, the loading of the model was performed through a nodal force applied at a height of the crown, of various amplitudes, $\mathrm{F}=1 \mathrm{~N} ; 2,3$ and $4 \mathrm{~N}$. The values of stress are: maximum stress of the whole structure and the shearing effect for the pulp.

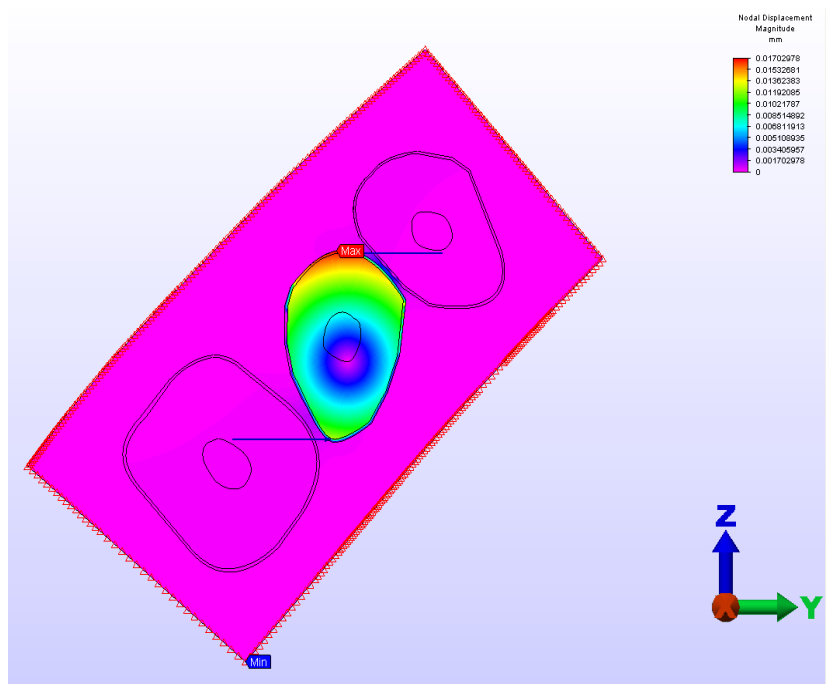

Figure 3. The model for teeth rotation simulation.

We analyzed the values of stress - Von Mises maximum stress/minimum stress with the effect of expansion/compression of the structure tissue and the shearing effect stress, relevant for the pulp. The structure modeled and studied is: first molar, premolar 1 and premolar 2, with all its constituents elements: dentine, pulp, periodontal ligament - alveolar bone.

The loading of the model was performed by a nodal force, with the amplitude of $300 \mathrm{~g}$, respectively $\mathrm{F}=3 \mathrm{~N}$, applied at a height of the tooth crown - corresponding to the various positions of the bracket, placed in the plane Oyz, on the vestibular or lingual side. It is considered the bracket action given by the nodal force $\mathrm{F}$ as a result of the forces distributed on the bracket-tooth contact surface. A twodimensional plane model was created, representing a median section on the height of the structure. The characteristics of the material are: Young's E modulus and Poisson's ratio.

The components of the modeled structure are:

Finite element numbers EF: 19852

Dentine Nr. EF: 5895

Pulp Nr. EF: 6751

PDL Nr. EF: 1035

Bone Nr. EF: 6138 
The elements of the structure are mainly stressed in: fiber compression in the direction of the moment's action given by the forces and fiber stretching in the opposite direction of the action.

For the reproduction of a situation as close as possible to the real one, the loading of the model was performed through a nodal force applied at a height of the crown corresponding to the position of the bracket, in the plane Oyz.

On the buccal/lingual surface, we apply forces of various amplitudes, progressively increasing: $100 \mathrm{~g}, 200 \mathrm{~g}$, 300 g, 400 g, respectively: $F=1 \mathrm{~N} ; F=2 \mathrm{~N} ; \mathrm{F}=3 \mathrm{~N} ; \mathrm{F}=4 \mathrm{~N}$.

\section{Results}

The characteristics of the material are Young's E modulus and Poisson's ratio of the components of the modeled structure.

The following values are relevant for the study:

Maximum principal Stress $\sigma 1 \max$ - maximum positive values with stretching effect on tissues (Figure 4).
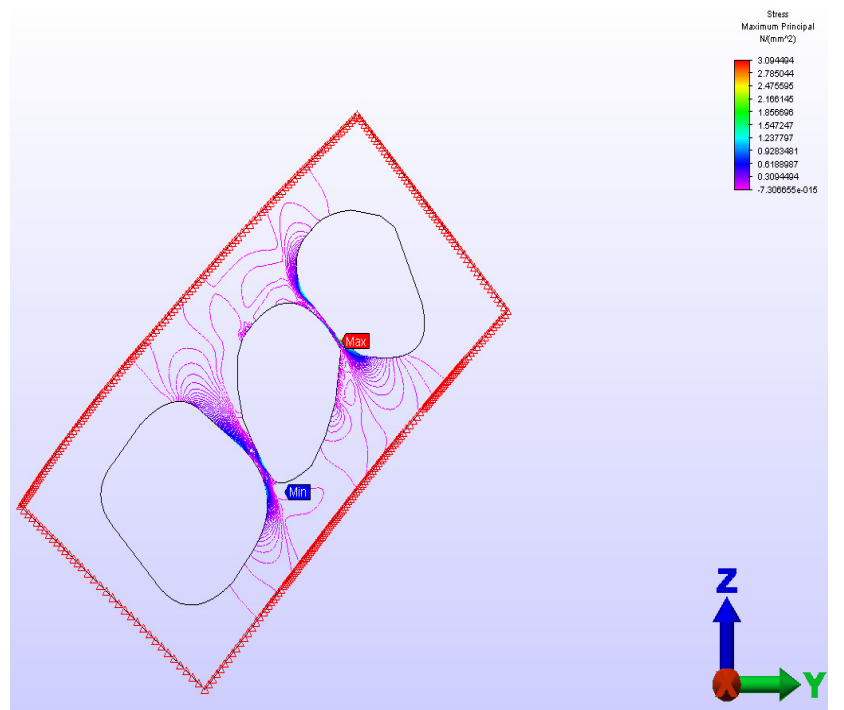

Figure 4. Stress Maximum Principal.

Minimum principal Stress $\sigma 2 \mathrm{~min}$ - maximum negative values with compression effect on tissues (Figure 5).

Shearing Stress $\tau$ yz, produces in the section plane Oyz - with great importance for the central component the pulp (Figure 6).

The most stressed elements of the structure are the pulp and the ligament, PDL revealed by von Mises stress. The elements of the structure are mainly stressed in fiber compression in the direction of the moment's action given by the orthodontic forces and in fiber stretching in the opposite direction. Out of the orthodontic movements: translation, tipping, intrusion-extrusion and rotation, the most dangerous was shearing stress (Figure 6).

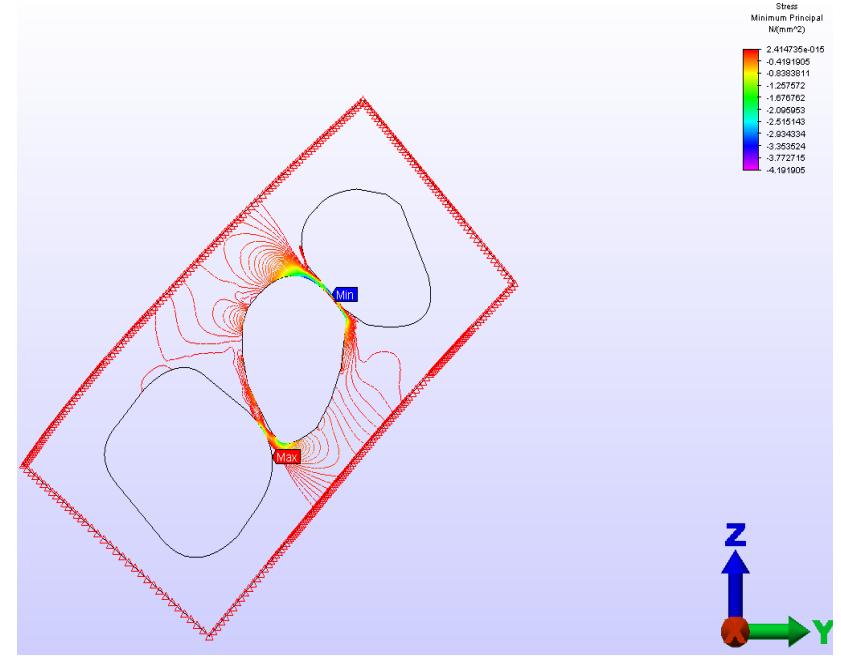

Figure 5. Stress Minimum Principal.

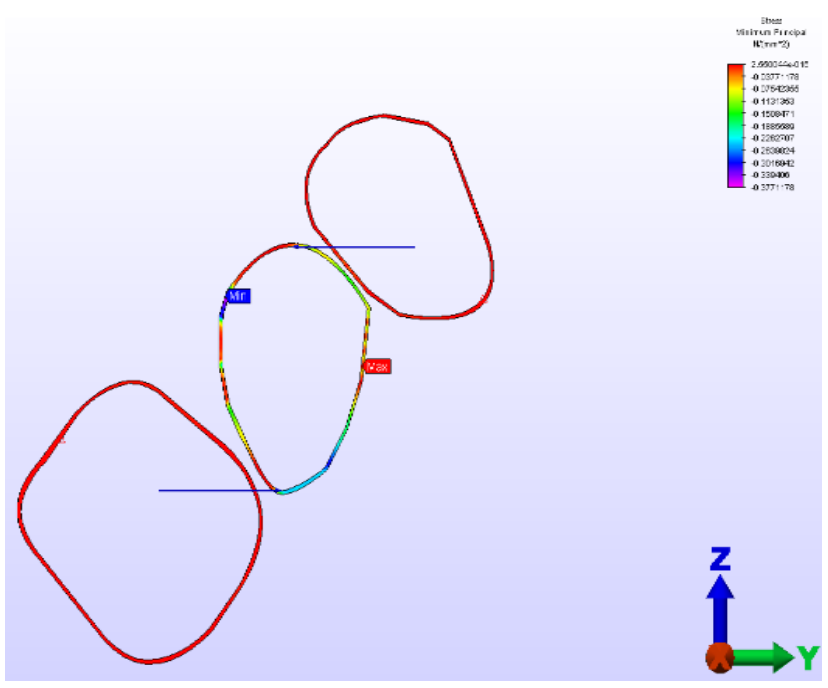

Figure 6. Shearing stress.

The maximum stress is on the second premolar as we can see in Figure 7.

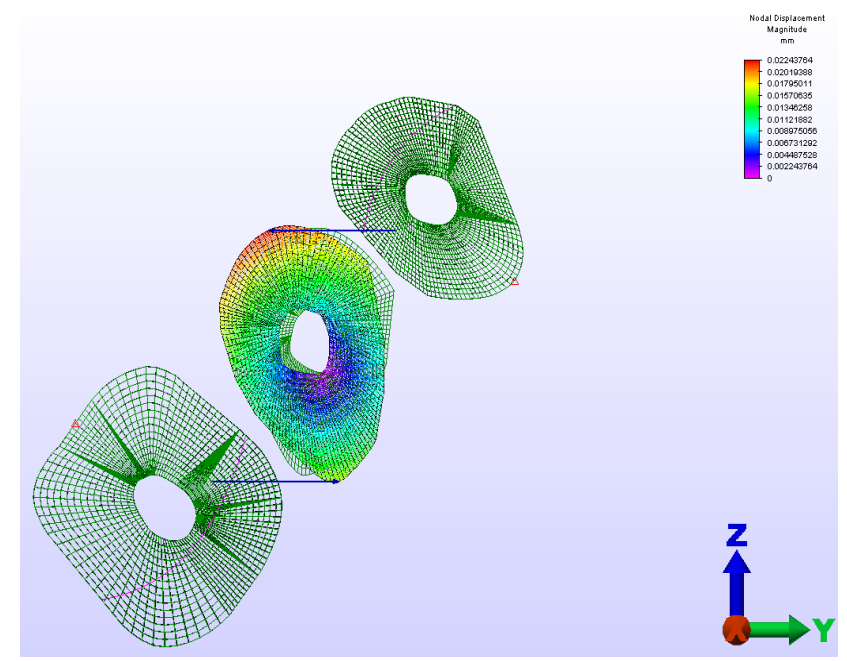

Figure 7. The rotation effect on the second premolar. 
It has been established that the maximum displacements are on the $2^{\text {nd }}$ premolar and is represented by: rotation/translation. In all elements of the structure, stress values are rising as the external load is increasing as we can see in table I.

Table I. Stress values in the elements of the periodontal structure.

The
components
of the $2^{\text {nd }}$
premolar

premolar

\begin{tabular}{lccc} 
& & o ech max & o 1 max \\
\multirow{3}{*}{ Pulp } & 1 & 1.739 & 1.19829 \\
& 2 & 2.39657 & 2.39657 \\
& 3 & 5.21701 & 3.59486 \\
Dentin & 4 & 6.95601 & 4.79315 \\
& 1 & 0.0055198 & 0.004807 \\
& 2 & 0.011039 & 0.009613 \\
\multirow{3}{*}{ PDL } & 3 & 0.0165596 & 0.0144203 \\
& 4 & 0.022075 & 0.019227 \\
& 1 & 1.04574 & 0.773623 \\
& 2 & 2.09148 & 1.54725 \\
Bone & 3 & 3.13723 & 2.32087 \\
& 4 & 4.18297 & 3.09449 \\
& 1 & 0.11347 & 0.10718 \\
& 2 & 0.226945 & 0.21436 \\
& 3 & 0.340417 & 0.32154 \\
& 4 & 0.453889 & 0.42872
\end{tabular}

If we compare the stress value, we can see that the stress von Mises are higher in the pulp (value 8) and in PDL is 4 (Figure 8, 9).

Stress values in the pulp are the highest compared to the other components. The values of the maximum stress in the pulp: equivalent Von Mises, Maximum principal Stress $\sigma 1$ max, Minimum principal Stress $\sigma 2 \mathrm{~min}$, are in the center of resistance. Maximum principal Stress $\sigma 1$ max values and Minimum principal Stress $\sigma 2$ min values are almost the same.

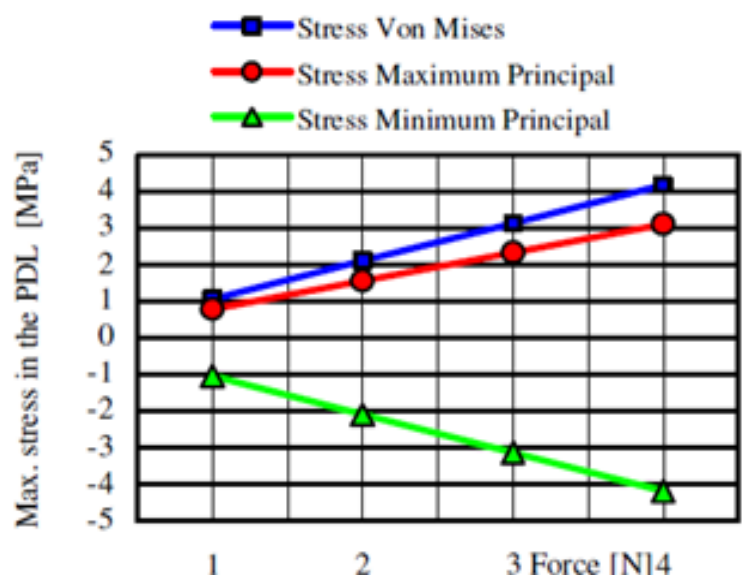

Figure 8. Stress in PDL during the rotation.

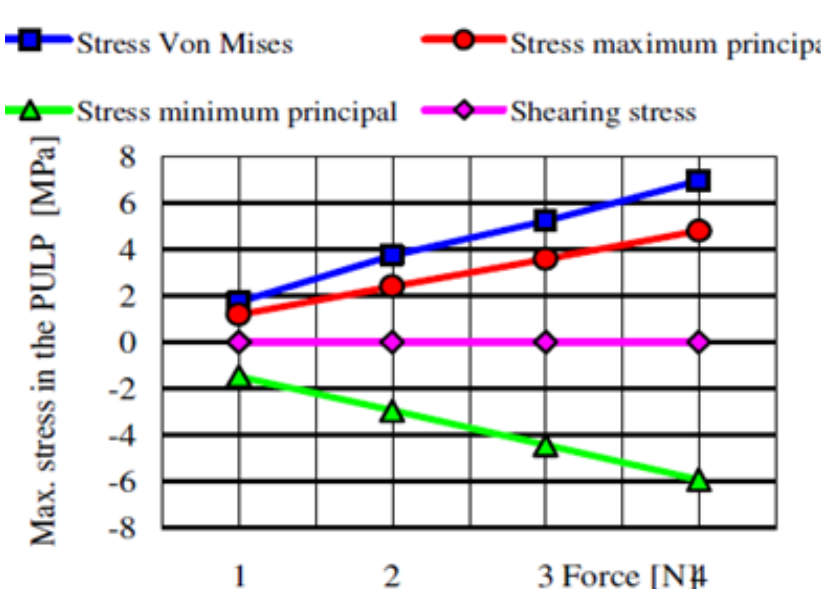

Figure 9. Stress in pulp during the rotation.

\section{Discussion}

Derotation of a tooth is considered to be a difficult orthodontic movement. Biomechanics theorists (Graber, Proffit) state that to produce a single tooth rotation around its axis, requires the force to reach magnitude much larger than those in order types of movements [4,5].

A rotation of the apex is difficult to achieve; this would entail the placement of the bracket very close to the alveolar crest - or even on the root of the tooth. The displacement of the node in the apex is negative - in the opposite direction of the force applied (there is a rotation of the tooth). The values of the displacements following the direction of the force $\delta y y$ are positive - stretching in the direction of the force applied - until $\mathrm{C}_{\mathrm{rot}}$, where $\delta \mathrm{yy}=$ 0 , then they become negative - compressed fibers.

The movement of the root in the alveolar bone at the apex level will be possible due to the extremely low rigidity of the periodontal ligament within its small thickness.

The displacement of the root is also possible by compressing the alveolar bone - a structure with higher rigidity, only in time, through a progressive application of the external force and determine a shearing stress in the pulp.

Our study demonstrates that the pulp stress distribution: equivalent Von Mises $\sigma_{\text {ech, }}$ maximum principal $\sigma_{1}$, minimum principal $\sigma_{2}$ are similar, with the maximum being in the zones close to the center of resistance and the decreasing of approximate values in a radial direction towards the exterior contour of the pulp [6,7].

Maximum values of the pulp stress: equivalent Von Mises $\sigma_{\text {ech }}$, maximum principal $\sigma_{1 \max }$, minimum principal $\sigma_{2 \min }$, are in the center of rotation and around it, this dangerous phenomenon can produce the destruction of the pulp in its center where the terminal nerves are, with the results being similar with the other literature studies of the specialty. 
This fact should be avoided in practice through the application of the principal force as close as possible to the center of resistance of the tooth [8].

Rotation movement provokes a torsion and an accumulation of fibers at the angles of rotation.

Relapses are due to collagen fibers of the periodontal ligament. These fibers that are composed of white collagen lack elastic properties however, and present an undulation capable of developing a replacing force whilst stretched. Relapses appear when a tooth was rotated/derotated much too fast with high continuous force $[7,9]$.

\section{Conclusions}

1. The highest stress values are in the Pulp. Similar values to the ones in the Pulp are in the PDL as well;

2. Shearing stress $\tau$ yz are in around the resistance center

3. The accumulated stress effect in the pulp becomes dangerous.

4. The orthodontic movements given by dental force values higher than $1.5-2 \mathrm{~N}$ are to be avoided.

5. There lies a real danger of shearing the pulp, at an increased, uncontrolled external action applied to the components of the bracket;

6. Compared to other orthodontic movements, the phenomena resulted from the rotation of the tooth are the most dangerous.

\section{References}

1. Rees JS, Hammadeh M. Undermining of enamel as a mechanism of abfraction lesion formation: a finite element study. Eur J Oral Sci. 2004;112:347-352.

2. Cattaneo PM, Dalstra M, Melsen B. The finite element method: a tool to study orthodontic tooth movement. J Dent Res. 2005;84:428-433.

3. Chun KJ, Yoo OS, Won YY, Lee JY. Morphological study and stress analysis of Korean Mandibular second premolar. Int J Precis Eng Manuf. 2009;10:141-145.

4. Nanda R, Kapila S. Current therapy in orthodontics. Mosby Elsevier; 2010: pp. 228-250.

5. Knox G, Jones ML, Hubsch P, Middleton J, Kralj B. An evaluation of the stresses generated in a bonded orthodontic attachment by three different load cases using the Finite Element Method of stress analysis. J Orthod. 2000;27:39-46.

6. Gurbuz T, Sengul F, Altun C. Finite element stress analysis of short-post core and over restorations prepared with different restorative materials. Dent Mater J. 2008;27:499-507.

7. Shahzad H. Mechanics of Orthodontic Tooth Movement. Final Year BDS. Roll No. 52,2014. Available from: https:// www.slideshare.net/ShahzadHussain3/mechanics-40889224

8. Choy K, Pae E, Park Y, Kim KH, Burstone CJ. Effect of root and bone morphology on the stress distribution in the periodontal ligament. Am J Orthod Dentofacial Orthop. 2000;117:98-105.

9. Rudolph DJ, Willes PMG, Sameshima GT. A finite element model of apical force distribution from orthodontic tooth movement. Angle Orthod. 2001;71:127-131. 